\title{
Safer Care: Shaping the Future
}

\author{
Liam Donaldson
}

\subsection{Introduction}

Understanding of, and commitment to, patient safety worldwide has grown since the late 1990s. This was prompted by two influential reports: To Err is Human [1] produced by the Institute of Medicine (now called the National Academy of Medicine) in the USA and An Organisation with a Memory [2] produced by the United Kingdom Government's Chief Medical Adviser. Both reports recognised that error was routine during the delivery of healthcare: affecting something like one in ten of all hospital patients. In a proportion of cases, the outcome produced was serious, even fatal.

The reports also drew attention to the poor performance of healthcare, as a sector, worldwide on safety compared to most other high-risk industries. Notably, aviation has shown remarkable and sustained improvements in the risk to passengers of air travel over four decades. Both reports called for greater focus on, and commitment to, reducing the risks of healthcare.

Since then, the quest to improve the safety of care for patients has become a global movement. Important bodies like the World Health Organization (WHO) [3], the Gulf Cooperation Council (GCC) [4], the Agency for Healthcare

L. Donaldson $(\bowtie)$

London School of Hygiene and Tropical Medicine, London, UK

e-mail: liam.donaldson@1shtm.ac.uk
Research and Quality (AHRQ) [5], the European Commission [6], and the Organisation for Economic Development (OECD) [7] have produced strategic documents, conducted studies, provided evidence and guidelines, initiated programmes of action, and galvanised the support of political and health leaders worldwide.

This has led to a remarkable transformation in the way that patient safety is viewed. Having been a subject of minority academic interest, it is now a firm priority for most healthcare systems.

Yet, the current state of patient safety worldwide is still a source of deep concern. As data on the scale and nature of errors and adverse events have been more widely gathered, it has become apparent that unsafe actions are a feature of virtually every aspect of healthcare. Furthermore, there is paucity of research on the frequency of errors and their associated burden of harm in areas such as primary care and mental health. Reports of the apparently avoidable deaths of patients regularly feature in media reports in many countries and undermine public confidence in the health services available to citizens. Moreover, many events recur with efforts to prevent them, on a large scale, proving ineffective. Expert commentators have explained this, in part, as being due to a punitive culture of individual blame and retribution holding back an approach that emphasises learning, not judgement as the route to improvement. 
In this chapter, I will reflect on some of perspectives in patient safety that the world of healthcare has adopted. These, and others, are dealt with in-depth in later chapters. I will also set out some of the key developments in the global level journey on patient safety.

\subsection{Thinking About Safer Healthcare}

By the end of the twentieth century, there was growing interest in avoidable adverse outcomes of healthcare from some clinical groups, researchers, and campaigners as well as victims of healthcare-induced harm and their families. The term used most widely at that time to describe such events was "medical error" [8]. It still is quite a common descriptor but the domain of healthcare that deals with risk of harm to patients and its prevention is now almost universally called "patient safety" [9].

In any complex system like a health service, human error, and mistakes - and hence adverse events - are inevitable. A programme to improve safety for patients cannot be based on eliminating error and mistakes - that would be impossible. A healthcare system, though, can reduce the occurrence of human error, minimise its impact on the patient when it does occur and learn so that actions can be taken to protect future patients.

\subsubsection{Accidents and Incidents: The Importance of Systems}

In exploring the reasons why things go badly wrong in healthcare, it becomes clear that its situation is not unique. There are many parallels with other sectors. Research and best practice experience outside the healthcare field has shown that safety comes down to appreciating that big improvements are not made by telling people to take care but by understanding the conditions that provoke error.

Extensive study in the non-health field has shown that with most unintended failures there is usually no single explanatory cause for the event.
Rather there is a complex interaction between a varied set of elements, including human behaviour, technological aspects of the system, sociocultural factors, and a range of organisational and procedural weaknesses $[10,11]$.

Wide scale systematic studies of these issues in healthcare are less common than in other highrisk industries, but available evidence suggests a similarly complex pattern of cause and effect relationship [12, 13].

Understanding the underlying reasons, or root causes, of why things go wrong is critical for success. The deeper causes of adverse patient incidents do, indeed, lie in the management and organisational systems that support the delivery of care. Research has shown that the causes are rooted in factors such as inadequate training, lack of communication, lack of information, faulty equipment, or poor physical environment. Asking staff to work in these conditions will risk causing harm to patients.

Building safety into health services by understanding the sources of risk within systems and eliminating them must be a core priority for all providers of healthcare (Table 5.1).

The key principle in safety generally (not just in healthcare), that unsafe systems provoke human error, is a different way of looking at the world, and requires a different philosophy of practice.

It was the introduction of experts from other fields that changed the way that healthcare looked at its own accidents and errors. No longer would

Table 5.1 Ten practical questions to ask about risk in a clinical service

\begin{tabular}{l|l}
\hline 1 & Describe the risks: what could go wrong? \\
\hline 2 & What is being done to manage the potential risks? \\
\hline 3 & What are the consequences if risks not managed? \\
\hline 5 & $\begin{array}{l}\text { Are the sources of the risks clinical, } \\
\text { organisational, or both? }\end{array}$ \\
\hline 6 & How often will the risks occur? \\
\hline 7 & Can you rate the risks' severity? \\
\hline 8 & $\begin{array}{l}\text { What level of control is there over the occurrence } \\
\text { of the risks? }\end{array}$ \\
\hline 9 & How will the reduction in risks be sustained? \\
\hline 10 & $\begin{array}{l}\text { How will you make all relevant staff aware of the } \\
\text { risks? }\end{array}$ \\
\hline
\end{tabular}




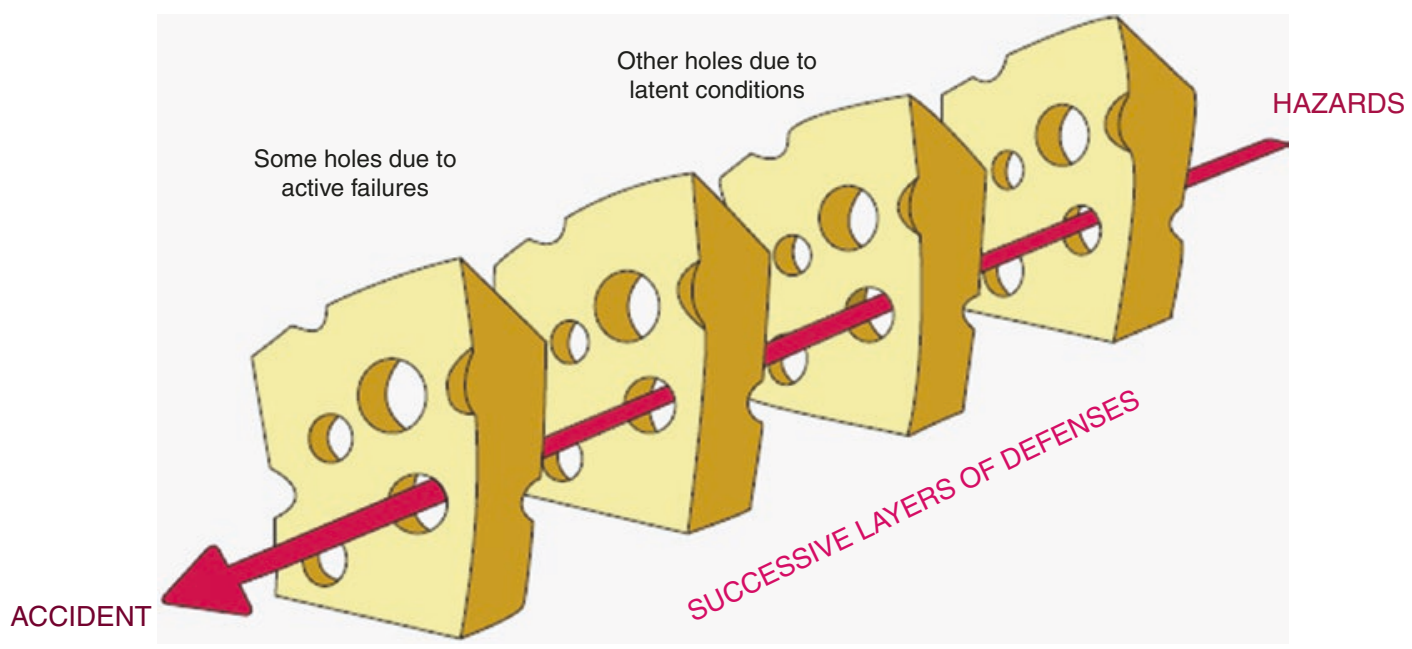

Fig. 5.1 The Swiss Cheese model of accident and incident causation. (Source: Professor James Reason by kind permission to the author)

an incident that killed or harmed a patient be seen as an unfortunate one-off local occurrence with no more general lessons to be learned. One of the major figures from outside healthcare to explain this perspective was Professor James Reason from the University of Manchester in England [14]. He put forward a compelling metaphor to encourage more broad-based thinking. He compared the risks of an accident or incident to the holes in slices of a Swiss cheese (Fig. 5.1). The solid pieces of cheese are the system's defences, whilst the holes are the weaknesses. The holes in the slices of James Reason's Swiss cheese-the organisation's system-open, close, and realign constantly. Some of the holes or risks are unsafe actions by individuals: slips, lapses of attention, mistakes, or violations of procedure. Many more are due to what Reason calls "latent conditions". These things like lack of training, weak procedures, and faulty or poorly maintained equipment create preconditions for failure.

Doctors traditionally have not been trained to think systemically. Their concern is the patient in front of them. They realise, of course, that their treatments and decisions can have negative outcomes, but their training puts these in the currency of "complications" or "side effects". The surgeon knows that her patient can develop postoperative bleeding. The physician knows that his drug can provoke a reaction. The surgeon though probably thinks less about the propensity for the system, through its design, to make it more likely that she will operate on the wrong side of the body. The physician ordering anticoagulants probably thinks more about blood tests and clinical monitoring data than the risk of a patient being given 15,000 units of heparin and killed when the intended dose was 1500 units but the abbreviation for "unit" was interpreted by the administering nurse as a zero.

Every day, around the world, patients die and are harmed because of these and similar circumstances. Human error occurs in weak systems: those that promote error rather than reducing its likelihood. Tomorrow's practitioners must not only think about themselves and their actions. They must also have "systems awareness".

It is also vital for health policymakers, healthcare leaders (not only clinical staff) to understand and embrace systems thinking. Frontline awareness of systemic weaknesses and risks is important but so too is strategic awareness by those responsible for the infrastructure, organisation, and delivery of care for communities and populations.

A system is sometimes a whole healthcare service. It is also a collection of processes of care within a health facility or care setting. In a large 
hospital, there may be 50 individual service groups all with their own processes and procedures. So, a systems perspective when something goes wrong, for example, can focus on the factors that led to a nurse inadvertently giving an infant a fatal overdose of a drug intravenously. Or, it might take an even broader view if the problem necessitates it. For example, an investigation of high healthcare infection rates might conclude that a group of African hospitals cannot maintain clean care because they do not have a source of clean water. As a result, finding a way to cheaply and locally manufacture an alcohol hand rub could help staff reduce infection rates. That would be aligning a systemic cause of harm with a systemic solution.

There are good examples of large-scale systemic actions led by clinicians. Global clinical networks of specialists and professional bodies are very well placed to identify common highrisk situations and galvanise support for action. The international clinical movement to reduce harm from sepsis [15] has shown how raising awareness and championing the need for action on a systemic patient safety issue can lead to change in attitudes and practice right across the world. Anaesthetic risk has been much reduced by combined research and action driven by organisations in this specialty either nationally, regionally, or globally.

\subsubsection{Culture, Blame, and Accountability}

The implications of system thinking in patient safety are quite profound. It means that ministries of health, managers of health facilities, the media, and the public must accept this paradigm as an explanation for the harm caused and cannot take a routinely "off with their heads" approach when something serious happens. Blaming individuals is common. It is easy, and generally popular. However, it is unfair, counter to developing a strong patient safety culture where learning benefits future patients. It has led many doctors and nurses who have simply made an honest mistake to end up behind bars. The force of public outrage is often too great for the chief executive officer of a hospital or health minister to withstand. Their principles are sacrificed and they take the easy way out. The damage to their leadership in the eyes of their staff is then incalculable. They did not have the courage to defend the learning culture when the chips were down.

This is one of the most difficult and debated areas of patient safety and is usually referred to as the "blame culture" principle. There are many other dimensions to considering culture in relation to patient safety and the goal of promoting, sustaining, and consistently delivering safer care (Fig. 5.2). Also within the culture of organisa-
Fig. 5.2 Patient safety culture has many strands (C) Sir Liam Donaldson)

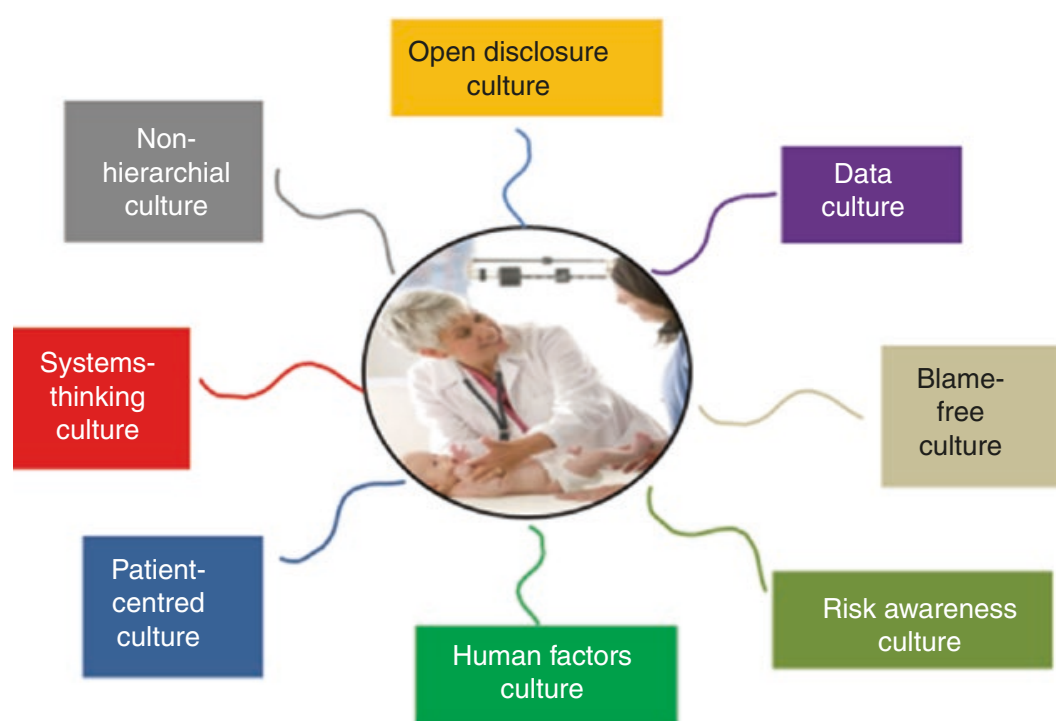


tions, there are certain behavioural aspects that will place patients at higher risk, including: for example, an arrogant belief that the organisation is too good to fail, a tendency to avoid dealing with signs that all is not well, hierarchical atti-

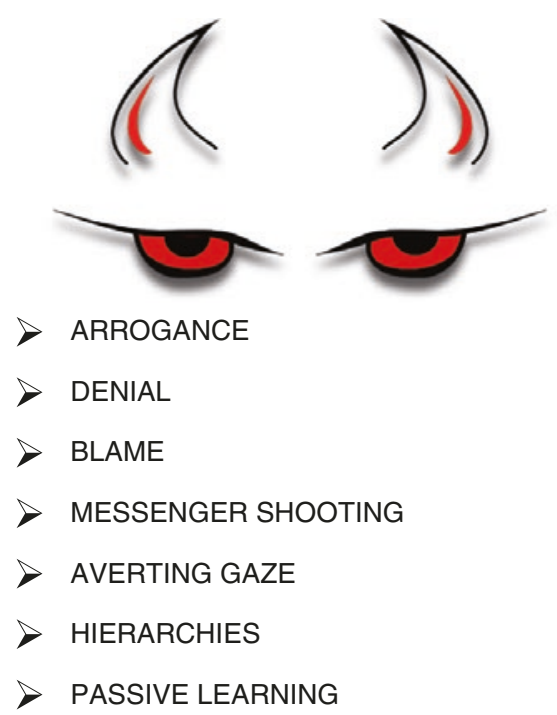

Fig. 5.3 Seven deadly sins: harmful behaviours within health organisations (ㄷ Sir Liam Donaldson) tudes where a junior nurse dare not challenge a senior doctor even if he is behaving unsafely, and ostracising whistle-blowers and others who are trying to highlight dangers (Fig. 5.3).

Modern healthcare is delivered in a complex, fast-moving environment. With the wrong culture, together with staff that are unaware of the potential risks of the care that they are delivering, then unsafe care may burst through and begin to kill and harm patients (Fig. 5.4).

\subsubsection{Leadership at the Frontline}

There is a caveat to an entirely systemic view of the world. Other high-risk industries do not set aside the need to focus on the individual as well as the system. This is not to blame them but to ensure that they are educated in risk and its importance, skilled, capable, and conscientious.

For example, in the airline industry, the number of times that an airline pilot might be assessed during their career could be as high as a hundred. Rehearsing in a simulator regularly, somebody flying with them in the flight deck, having regular
Fig. 5.4 The bulging triangle: how unsafe care can break out of its boundaries (ㄷ Sir Liam Donaldson)

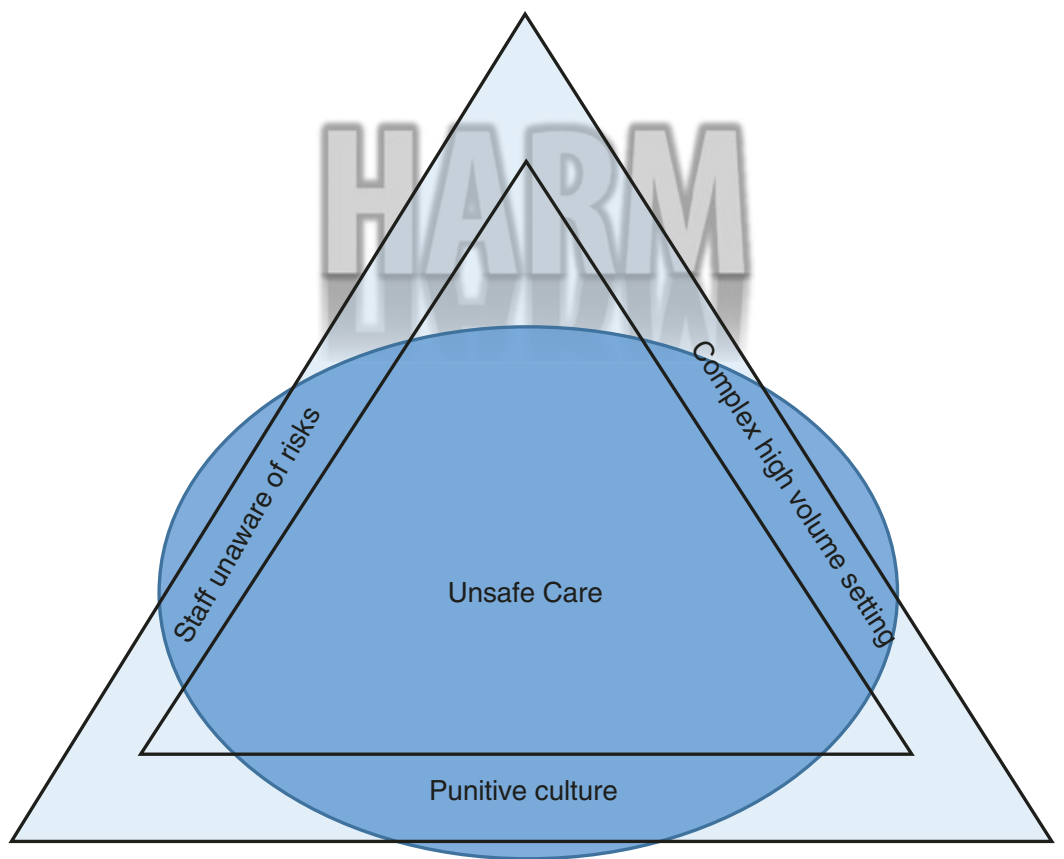


medicals, these are part of the process of ensuring safe air travel. In many parts of the world, once a doctor has finished training, they may not have any regular checks on their performance or challenges to how they would handle emergency situations. Simulation is playing an increasingly important part in healthcare, particularly in education and training. Other industries are much further ahead in simulating unsafe situations and training their staff. It is an exciting idea to develop skills, away from the patient and then bring the practitioner to the patient when they have a higher level of skill. It is not the whole solution to creating "safety-wise" practitioners.

One of the great strategic needs in patient safety is for leadership, and role models in patient safety for young practitioners. There are many wonderful patient safety leaders at global level and within countries. They have been instrumental in making patient safety the priority that it is today within health systems around the world. However, there are far too few of them. Every clinical team in every part of every health system of the world needs skilled committed leadership in patient safety. This is needed because every patient must be protected from the ever-present risk of harm. It is here that we can look to the young generation of doctors, nurses, and other health professionals who are already demonstrating their interest and passion for patient safety.

\subsection{Global Action to Improve Safety}

Through the early years of the twenty-first century, patient safety began to feature as a priority or programme of work in larger hospitals in the higher income countries of the world, and in some national health systems. It was still a long way from the mainstream of healthcare leaders, policymakers, and frontline clinical staff. Initially, it was a subject very much in the domain of a small number of thought leaders, researchers, and enthusiasts. Moving these deliberations and debates to global level catalysed action in country health systems on a much more extensive basis and served a convening function by bring- ing health leaders, politicians, experts, researchers, and patient representatives into the same rooms.

\subsubsection{Patient Safety on the Global Health Agenda}

The World Health Organization (WHO), the United Nations agency responsible for health, first raised the profile of patient safety to global importance. In May 2002, the 55th World Health Assembly (the annual policy-making meeting of all 192 countries of the world) adopted Resolution 55.18. This urged Member States to pay the closest possible attention to the problem of patient safety and to establish and strengthen science-based systems necessary for improving patient safety and the quality of healthcare [16].

Following this, in May 2004, the 57th World Health Assembly supported the creation of an international alliance to facilitate the development of patient safety policy and practice in all member states, to act as a major force for improvement globally. The World Alliance for Patient Safety, a partnership between WHO and external experts, healthcare leaders, and professional bodies, was launched formally in October of 2004.

\subsubsection{World Alliance for Patient Safety: Becoming Global}

The World Alliance for Patient Safety formulated an initial programme of work framed as a series of six important actions intended to reduce harm caused to patients:

- The first Global Patient Safety Challenge, focusing, on the theme of healthcareassociated infection [17]

- A Patients for Patient Safety network involving patient organisations and led by individuals who had suffered avoidable harm from healthcare [18]

- A Taxonomy for Patient Safety, ensuring consistency in the concepts, principles, norms, and terminology used in patient safety work [19] 
- A Research for Patient Safety initiative to identify priorities for patient safety-related research in high-income, middle-income, and low-income countries as well as projects and capacity building particularly aimed at lowincome countries [20]

- A Solutions for Patient Safety programme to identify, develop, and promote worldwide interventions to improve patient safety

- A set of Reporting and Learning best practice guidelines to aid in the design and development of existing and new reporting systems [21].

The overall aims of this global partnership for patient safety were: to promote the development of evidence-based norms for the delivery of safer patient care, to create global classifications for medical errors, and to support knowledge sharing in patient safety between member states. There was also a strong advocacy role to raise awareness of the risks of unsafe care and generate a better understanding of the reasons why harm occurs, to draw attention to the most effective preventive measures, as well as establishing the means to evaluate them.

At the outset, there were three core principles that underpinned the initial focus for action at global level:

- A commitment to placing patients at the centre of efforts to improve patient safety worldwide

- A focus on improving ways to detect and learn from information about patient safety problems within and across countries (with a particular emphasis on methods and tools for detecting patient safety problems in lowincome countries)

- A need to build up the knowledge base of interventions which have been shown to help solve patient safety problems, together with a more rapid and systematic dissemination of information worldwide on successful strategies.

The World Alliance for Patient Safety, in its publications, its events, and when its members spoke at conferences, always sought to educate and inform about the concepts and philosophy that should underlie a modern approach to safety in healthcare.

\subsubsection{The Global Patient Safety Challenges}

As each of the foundation strands of the global patient safety initiative began to be implemented, they attracted a great deal of interest, involvement, and began to shape change in healthcare systems around the world.

At the beginning, it had been important to choose a major aspect of patient safety that affected all countries of the world and was big enough to warrant intensive action on a global scale. Healthcare infection fitted these criteria immediately. It was endemic within every healthcare system. In high-income countries, there was great concern, not just about the persistence of the problem, but the emergence of life-threatening antimicrobial-resistant strains such as methicillinresistant Staphylococcus aureus (MRSA). In low-income and middle-income countries, the problem was even more serious especially where the infrastructure of care was weak.

The first Global Patient Safety Challenge, aimed to engage the world's health systems in a movement to reduce healthcare infection. It began by convening all the leading experts to formulate ground breaking new evidence-based guidelines on hand hygiene. In addition, a major study was mounted to assess the burden of healthcare infection (particularly in low- and middleincome countries). This first Challenge Clean Care is Safe Care [17] invited health ministers to personally, and publicly, sign a pledge to address healthcare infection in their countries.

The first Global Patient Safety Challenge was the flagship element of the World Alliance for Patient Safety's initiative. It was highly visible and easily understood by politicians, health professionals, and the public. It was relevant to all countries: rich, poor, and emerging economies. Everyone had a vested interest in its success because anyone could need treatment in a health 
facility and could therefore become the victim of harm by acquiring an infection.

In driving forward Clean Care is Safer Care, a wide range of supporting activities and campaigns was implemented. The idea of this Challenge generated huge interest and enthusiasm across all six WHO regions. As ministers signed their pledges in country and regional launches and events, from a small start, eventually, the commitments covered $85 \%$ of the world's population.

The WHO hand hygiene global campaign (SAVE LIVES: Clean Your Hands) [22] launched in 2009 has been particularly successful. Before the Challenge, alcohol-based hand rubs (hand sanitisers) were not commonplace in hospitals around the world. The core message was that the lack of consistent, immediate, access to a sink equipped with soap and single-use towels (highincome countries) and/or the unavailability of clean water (many low-income countries) put patients at risk. The evidence of higher efficacy, effectiveness, and skin tolerability of alcoholbased hand rubs made them the method of choice to assure hand hygiene. The Challenge made alcohol hand rubs more affordable to the poorest hospitals of the world by ensuring that the University Hospital of Geneva formulation became available with no patent restriction for local manufacture.

A further key step in achieving the global reach of the hand hygiene programme was the development of the Five Moments for hand hygiene model [23]. This emphasised the points in the process of patient care when the risks of transmission of an infection by a caregiver's hands were highest. The Five Moments' visual image (Fig. 5.5) is striking and easily remembered by frontline staff; therefore, it has acted as a technical educational tool that succeeded in standardising practice worldwide but also it has become a brand of safety with global spread.

Overall, the first Global Patient Safety Challenge represented a proven change model that mobilised the world around infection prevention through: (a) awareness raising about the burden of the problem to engage stakeholders; (b) an approach to engage nations through demonstrable commitment; (c) the availability of evidencebased guidance and implementation tools to drive improvement.

The original concept of such a Challenge was of a 2-year start-up period, after which responsibility for its continuance would pass to WHO member states and their healthcare systems. However, Clean Care is Safer Care generated so
Fig. 5.5 Five moments for hand hygiene. (Source: World Health Organizationreproduced with permission)

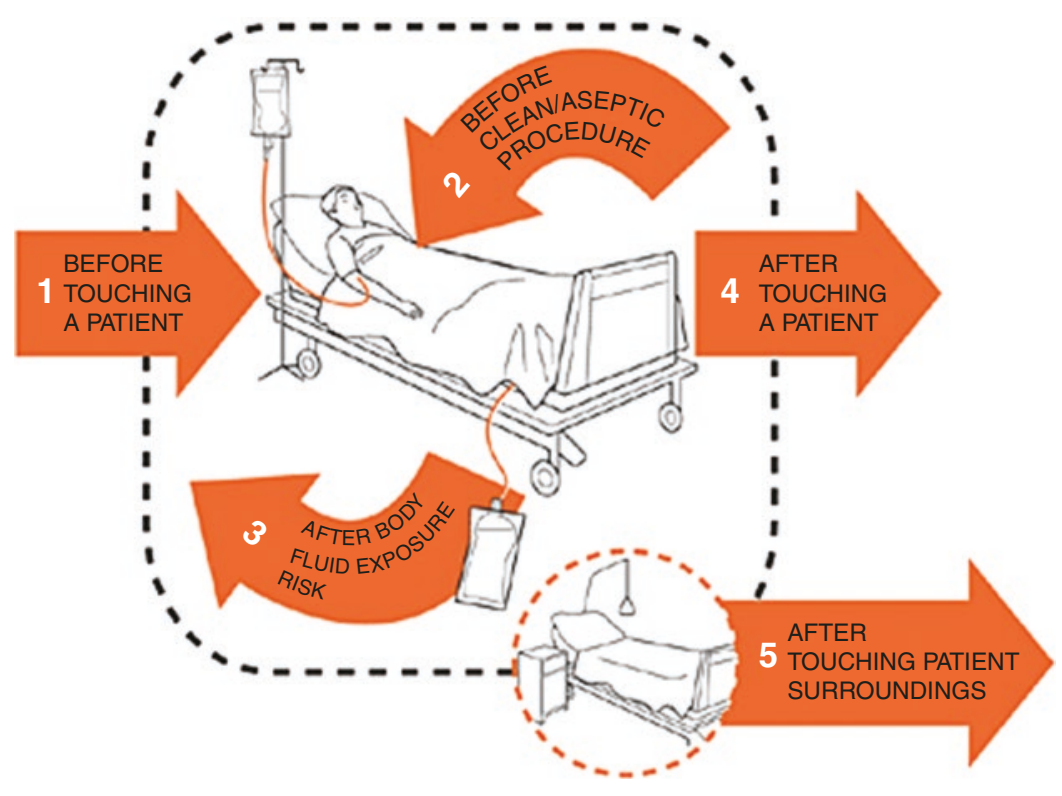


much momentum, passion, and so great a sense of solidarity across the world that the WHO's team in Geneva was continuing to play a strong leadership role 10 years after the launch. This success and the perception of the need for supporting infection prevention and control improvement in many countries, led the WHO to institute a new, formalised infection prevention and control global unit.

Other important achievements of the first Challenge and associated global infection prevention and control work included:

- An assessment of the burden of healthcare infection in low- and middle-income countries

- WHO guidelines and 100s of associated publications

- Fifty-five hospital departments across six countries demonstrated scientifically successfully implemented a hand hygiene multimodal improvement strategy

- Over 30 countries established WHO-guided local production of alcohol-based hand rub

- Over 50 countries ran successful hand hygiene national campaigns

- Almost 20,000 health facilities in 177 countries joined the WHO SAVE LIVES: Clean Your Hands campaign

- Global initiatives and engagement of thousands of health workers around hand hygiene every year on 5th May

- Patient engagement/information tools issued

- Reports from seven global surveys, on hand hygiene and a range of infection prevention and control and antimicrobial resistance priorities

- Hand hygiene and infection prevention and control messages embedded in key programmes of work including antimicrobial resistance, WASH and maternal and child health

- Alcohol-based hand rub featured in the WHO List of Essential Medicines

- Guidance produced on infection prevention and control during the 2014-15 Ebola virus disease outbreak (through the leadership of the team)
- New evidence-based guidelines on injection safety and ongoing testing of an implementation campaign in three countries supported by more than 20 new tools

- New evidence-based guidelines for the prevention of surgical site infections based on 27 systematic literature reviews and including 29 recommendations

- New evidence-based guidelines on the core components of effective infection prevention and control programmes to reduce harm from health care-associated infections and antimicrobial resistance

A second Global Patient Safety Challenge recognised the relatively high burden of disease arising from unsafe surgical care. Safe Surgery, Saves Lives [24] created a surgical checklist that was piloted, evaluated, and promoted for use globally. Initial evaluations showed that the checklist reduced morbidity and mortality associated with surgery in early studies of its use. Major professional bodies across the world endorsed it. It is in widespread use in hospitals in many countries and, increasingly, it is seen as essential if the key risks of surgery are to be avoided. However, the original checklist has been widely adapted whilst the experience of the surgical checklist's use worldwide has not been formally revisited since its launch.

The checklist concept was developed further with the creation of the WHO Safe Childbirth Checklist [25], which focuses on reducing risk and adverse outcomes related to childbirth for both mothers and babies. Of the more than 130 million births occurring each year, an estimated 303,000 result in the mother's death, 2.6 million in stillbirth, and another 2.7 million in a newborn death within the first 28 days of birth. The majority of these deaths occur in low-resource settings and most could be prevented. The WHO Safe Childbirth Checklist supports the delivery of essential maternal and perinatal care practices and addresses the major causes of maternal death, intra-partum-related stillbirths, and neonatal deaths. The Safe Childbirth Checklist Collaboration has already made significant strides to improving maternal and neonatal 
health. It is hoped that the Checklist can become an effective life-saving tool that can be used in a wide range of settings.

\subsubsection{Patients and Families: Championing Change}

In addition to the expert reports that had drawn the attention of policymakers to problem of unsafe care, a powerful driving force for change was the visibility of tragic and harrowing situations in which patients had suffered serious harm or died. Some of the victims of this unsafe care, or often surviving family members, had risen above their personal tragedy to tell their stories very publicly and call for the world's healthcare systems to take action.

The World Alliance for Patient Safety established the Patients for Patient Safety Programme as one of its first actions. Susan Sheridan (a contributor to this book), whose son suffered brain damage, and whose husband died, both associated with medical error, was the first external lead of this programme. Over time, a global network of patient champions was established. Many were themselves victims of avoidable harm or they were a parent of a child who had died or had been harmed.

With the expanding ageing population, the rise in non-communicable diseases and everrising healthcare costs, there is more willingness than ever by healthcare providers to engage with patients, families, and communities. Recognising these challenges and opportunities, the Patients for Patient Safety Programme has restructured its approach to emphasise four key strategic objectives:

- Advocacy and awareness raising

- Capacity development and strengthening
- Partnerships with healthcare providers and policymakers

- Influencing and contributing to policy and research priorities

The Patients for Patient Safety network now has over 500 advocates, also known as Patients for Patient Safety champions, in 54 countries. Newsletters are produced quarterly to promote the sharing of knowledge and experiences.

The champions involved in the Patients for Patient Safety Programme have: acted as advocates for the importance of tackling unsafe care in the healthcare systems of their countries; participated in education and training programmes for healthcare professional staff; supported other victims of harm who have contacted them; and, served on boards and advised hospitals on the design of their services.

The role of patients and family members in the quest for safer healthcare worldwide has been of incalculable benefit to the advancing the case of patient safety globally in the last decade (Table 5.2). Their experience, wisdom, and courage has fuelled a journey whose eventual endpoint will be a coalescence of compassion and learning to eradicate serious harm from every healthcare system in the world.

\subsubsection{African Partnerships for Patient Safety}

African Partnerships for Patient Safety (APPS) [26] was launched in 2009. It was designed to fill a perceived gap in patient safety in Africa. It was part of a WHO response to the commitment to strengthen patient safety articulated by 46 ministries of health at the 58th session of WHO's Regional Committee for Africa in 2008.

Table 5.2 Value of involvement of patients and families who have suffered harm

\begin{tabular}{l|l}
\hline Role & Benefit \\
\hline Educator & Reinforces professional values of caring, compassion, and respect \\
\hline Storyteller & Wins hearts and minds of leaders and frontline staff; stays in the memory \\
\hline Advocate & $\begin{array}{l}\text { Gains commitment at wider political, public, and professional levels; initiates campaigns for specific } \\
\text { actions (e.g. for sepsis, for in-patient suicide) }\end{array}$ \\
\hline Partner & Strengthens design and delivery of future care pathways and patient safety programmes \\
\hline Reporter & Highlights new risks and improvement opportunities \\
\hline
\end{tabular}


African Partnerships for Patient Safety developed a multi-country, hospital-to-hospital partnership programme. Initial support came from the United Kingdom Department of Health. Subsequently, the Government of France funded expansion of the programme beyond English speaking countries. During the period 20092014, African Partnerships for Patient Safety oversaw the implementation of 17 hospital-tohospital partnerships. The partnerships comprised European hospitals from three countries (France, Switzerland, and the United Kingdom) and hospitals in 17 different countries in the WHO African Region (Benin, Burkina Faso, Burundi, Cameroon, Côte d'Ivoire, Ethiopia, Ghana, Malawi, Mali, Niger, Rwanda, Senegal, Togo, Uganda, the United Republic of Tanzania, Zambia, and Zimbabwe). Linguistic diversity was maintained through the involvement of English, French, and Portuguese speaking countries.

As African Partnerships for Patient Safety evolved, south-south patient safety partnerships were established between hospitals in Zimbabwe and between Morocco and its partnership hospital further south. In addition, a partnership was established involving the Johns Hopkins University Armstrong Institute for Patient Safety \& Quality and institutions in three African countries (Liberia, South Sudan, and Uganda).

African Partnerships for Patient Safety received widespread international attention and recognition. It illustrated how teams skilled in infection prevention and control and patient safety can act as a bridge between diseasespecific programmes and health systems. This strengthens interaction at the health facility level. It provided a very tangible entry point for broader improvement in service delivery. Evaluation of the programme showed gains in hand hygiene compliance by health workers, implementation of the WHO Surgical Safety Checklist, training and education of healthcare workers, medication safety, healthcare waste management, clinical audit, teamwork, and leadership.

A defining feature of the African Partnerships for Patient Safety approach is that it presented an alternative to traditional vertical, expert-driven, technical assistance improvement models. It used frontline expertise from across both arms of the partnership hospitals with a focus on codevelopment and relationship building. The tools developed by the programme are now being utilised across the world, notably through the United Kingdom's Department for International Development Health Partnerships Scheme, hospital partnership initiatives led by Expertise France, partnerships supported by the Ministry of Foreign Affairs of Japan, partnerships supported by the Tuscany region of Italy and a recent major initiative focused on hospital partnerships initiated by the Ministry of Health in Germany.

African Partnerships for Patient Safety illustrated how frontline passion and energy has driven implementation of patient safety initiatives through strong human interaction and solidarity across continents. The work has informed national policy direction in multiple countries in the WHO Region of Africa. Importantly, African Partnerships for Patient Safety has shone the light on the potential for high-income countries to learn from low-income countries, the so-called reverse innovation.

The work of African Partnerships for Patient Safety has provided a strong foundation for the development of a wider international effort on "twinning partnerships for improvement". This is particularly relevant given the increasing importance placed on quality as part of the fabric of Universal Health Coverage-driven reform processes across the world, and in particular in lowincome countries.

\subsubsection{Third Global Patient Safety Challenge: Medication Without Harm}

The World Health Organization (WHO) launched its third Global Patient Safety Challenge in 2016 [27]. Its aim is to reduce the global burden of iatrogenic medication-related harm by $50 \%$ within 5 years. The intention is to match the global reach and impact of the WHO's two earlier Global Patient Safety Challenges, Clean Care is Safer Care and Safe Surgery Saves Lives. The third Challenge, 
Medication Without Harm, invites health ministers to initiate national plans addressing four domains of medication safety, namely: engaging patients and the public; medicines as products; education, training, and monitoring of healthcare professionals; and systems and the practices of medication management (Fig. 5.6). It also commits the WHO to use its convening and coordinating powers to drive forward a range of global actions. A tool to empower patients is already available.

Three key areas of medication safety have been identified as early priorities. They will be the most visible and public-facing aspects of this latest Challenge, just as hand hygiene and the surgical checklist were the flagship elements of the first two Global Patient Safety Challenges. They are: high-risk medicine situations; polypharmacy; and transitions of care. Each is associated with a substantial burden of harm and, if appropriately managed, could reduce the risk of harm to large numbers of patients in health systems across the world.

\subsubsection{The 2019 WHA Resolution and World Patient Safety Day}

Further impetus and fresh momentum was injected into the global patient safety movement in 2019 when the World Health Assembly again considered patient safety. This came at a time when, despite efforts of the previous decade, harm due to unsafe care was recognised as one of the 10 leading causes of morbidity and mortality globally, exceeding malaria and tuberculosis and level with HIV.

In May 2019, the 72nd World Health Assembly designated patient safety as a global health priority; adopted resolution WHA72.6 [28] and established an annual World Patient Safety Day. WHA72.6 requests the WHO's Director General: "To emphasize patient safety as a key strategic priority in WHO's work across Universal Health Coverage agenda", and: "To formulate a global patient safety action plan in consultation with Member States, regional economic integration
Fig. 5.6 Third Global Patient Safety Challenge: strategic framework (C) World Health Organization 2018. Some rights reserved. This work is available under the $\mathrm{CC}$ BY-NC-SA 3.0 IGO licence)




organisations and all relevant stakeholders, including in the private sector".

This major commitment and the delivery of a comprehensive action plan will drive the shape of patient safety programmes across the world for the next decade.

\subsection{Conclusions}

In an era when the human genome has been mapped, when air travel is safer than ever before, and when information flows across the globe in seconds, patients cannot be reassured that they will not die because of weaknesses in the way that their care is organised and delivered.

Despite the extensive work that has been put in at global level and in health systems around the world, a sustainable model for safe healthcare is not in place.

Firstly, the scale of the problem is so great that it can no longer just be left to special interest and to advocacy. The ownership of the problem of patient safety needs to be everybody's business. The action to tackle it needs to be everybody's business.

Secondly, this has been going on for just too long. There can be no other high-risk industry with such a poor record in improving known areas of risk.

Thirdly, the WHO and other global agencies and leaders are calling on the 194 countries of the world to implement a policy of Universal Health Coverage. It is essential that health systems are built with patient safety and quality of care as their organising principle. Almost everyone who accesses healthcare will at some point be treated. That treatment needs to be safe. What stronger connection could there be between patient safety and universal health coverage?

\section{References}

1. Kohn K, Corrigan J, Donaldson M. To err is human. Washington, DC: National Academy Press; 2000.

2. Chief Medical Officer. An organisation with a memory: a report on learning from adverse events in the NHS. London: The Stationery Office; 2000.
3. Donaldson LJ, Fletcher MG. The WHO world alliance for patient safety: towards the years of living less dangerously. Med J Aust. 2006;184(10):S69-72.

4. Siddiqi S, Elasady R, Khorshid I, Fortune T, Leotsakos A, Letaief M, et al. Patient Safety Friendly Hospital Initiative: from evidence to action in seven developing country hospitals. Int J Qual Health Care. 2012;24(2):144-5.

5. Agency for Healthcare Research and Quality (AHRQ). Advances in patient safety: from research to implementation. 05-0021. CD ROM. Bethesda: AHRQ; 2005.

6. European Commission (EC). Report on the member states' implementation of council recommendations on patient safety. Brussels: EC; 2012.

7. Organisation for Economic Development and Cooperation (OECD). Measuring patient safety: opening the black box. Paris: OECD; 2018.

8. Leape LL. Error in medicine. J Am Med Assoc. 1994;272:1851-7.

9. Lilford R, Stirling S, Maillard N. Citation classics in patient safety research: an invitation to contribute to an online bibliography. Qual Saf Health Care. 2006;15(5):311-3.

10. Macrae C. Close calls: managing risk and resilience in airline flight safety. Basingstoke: Palgrave Macmillan; 2014.

11. Strauch B. Investigating human error: incidents, accidents, and complex systems. Burlington, VT: Ashgate; 2002.

12. Smetzer JL, Cohen MR. Lessons from the Denver medication error/criminal negligence case: look beyond blaming individuals. Hosp Pharm. 1998;33:640-57.

13. Toft B. External inquiry into the adverse incident that occurred at Queen's Medical Centre, Nottingham 4th January 2001. London: Department of Health; 2001.

14. Reason J. Managing the risks of organisational accidents. Aldershot: Ashgate; 1997.

15. Reinhart C, Daniels R, Kissoon N, Machad FR, et al. Recognising sepsis as a global health priority: a WHO resolution. N Engl J Med. 2017;377:414-7.

16. World Health Organization (WHO). World Health Assembly resolution WHA 55.18. Geneva: WHO; 2002.

17. Pittet D, Donaldson L. Challenging the world: patient safety and health care-associated infection. Int J Qual Health Care. 2006;18(1):4-8.

18. World Health Organization (WHO). Patients for patient safety: the London declaration. Geneva: WHO; 2006.

19. World Health Organization (WHO). The final technical report for the conceptual framework for the international classification for patient safety. Geneva: WHO; 2009.

20. Bates DW, Larizgoitia I, Prasopa-Plaizier N, Jha AK, Research Priority Setting Working Group of the WHO World Alliance for Patient Safety. Global priorities for patient safety research. BMJ. 2009;338:b1775. 
21. World Alliance for Patient Safety. WHO draft guidelines for adverse event report and learning systems: from information to action. Geneva: WHO; 2005.

22. Allegranzi B, Storr J, Dziekan G, et al. The first global patient safety challenge "Clean care is safer care": from launch to current progress and achievements. J Hosp Infect. 2007;65(Suppl. 2):115-23.

23. Pittet D, Allegranzi B, Sax H, Dharan S, PessoaSilva CL, Donaldson LJ, et al. Evidence-based model for hand transmission during patient care and the role of improved practices. Lancet Infect Dis. 2006;6(10):641-52.

24. World Alliance for Patient Safety. The second global patient safety challenge: safe surgery saves lives. Geneva: World Health Organization; 2008.
25. World Health Organization (WHO). Safe childbirth checklist collaboration: improving the health of mothers and neonates. Geneva: WHO; 2014.

26. Syed SB, Gooden R, Storr J, et al. African partnerships for patient safety: a vehicle for enhancing patient safety across two continents. World Hosp Health Serv. 2009;45:24-7.

27. Donaldson LJ, Kelley ET, Dhingra-Kumar N, Kieny M-P, Sheikh A. Medication without harm: WHO's third global patient safety challenge. Lancet. 2017;389(10080): 1680 .

28. World Health Organization (WHO). Resolution WHA 72.6: global action on patient safety. Geneva: WHO; 2019.

Open Access This chapter is licensed under the terms of the Creative Commons Attribution 4.0 International License (http://creativecommons.org/licenses/by/4.0/), which permits use, sharing, adaptation, distribution and reproduction in any medium or format, as long as you give appropriate credit to the original author(s) and the source, provide a link to the Creative Commons license and indicate if changes were made.

The images or other third party material in this chapter are included in the chapter's Creative Commons license, unless indicated otherwise in a credit line to the material. If material is not included in the chapter's Creative Commons license and your intended use is not permitted by statutory regulation or exceeds the permitted use, you will need to obtain permission directly from the copyright holder. 